\title{
Physical Properties Comparison of rGO-like Phase Prepared from Coconut Shell and the Commercial Product
}

\author{
Retno Asih*, ${ }^{1,2}$ Maya Mahirotul Septya, ${ }^{1}$ Erik Bhekti Yutomo, ${ }^{1}$ Fahmi Astuti, ${ }^{1,2}$ Malik Anjelh Baqiya, ${ }^{1}$ and Darminto*1,3 \\ ${ }^{I}$ Department of Physics, Faculty of Science and Data Analytics, Institut Teknologi Sepuluh Nopember (ITS) \\ Kampus ITS Sukolilo, Surabaya 60111, Indonesia \\ ${ }^{2}$ Meson Science Laboratory, RIKEN Nishina Center, Wako, Saitama 351-0198, Japan \\ ${ }^{3}$ Center of Excellence in Automotive Control and System, Institut Teknologi Sepuluh Nopember (ITS) \\ Kampus ITS Sukolilo, Surabaya 60111, Indonesia
}

\begin{abstract}
Electrical dan magnetic properties of graphene-derivatives materials are strongly influenced by their physical properties. Here we report a study on physical properties of reduced graphene oxide (rGO) prepared from two different raw materials, namely coconut shell (rGO-s) and graphite mineral (rGO-c, produced by Graphenea Inc.). rGO-s was prepared by carbonization method followed by mechanical exfoliation. While both samples have the same density of about $1.9 \mathrm{~g} / \mathrm{cm}^{3}, \mathrm{rGO}-\mathrm{c}$ has more porous compared to rGO-s. Specific surface area in rGO-c was also obtained much larger than that of rGO-s. Examinations on particle size and surface morphology show that rGO-c has homogenous particles which consist of transparent thin sheets, while rGO-s has rather heterogenous particles that look like dens stacked sheets. The presence of $\mathrm{C}$ and $\mathrm{O}$ was confirmed at the observed morphology. The difference in physical features was found to influence the obtained electrical conductivity of the samples. rGO-c has higher conductivity than rGO-s. Estimation on gap energy $\left(\mathrm{E}_{g}\right)$ indicates that $\mathrm{rGO}-\mathrm{c}$ and $\mathrm{rGO}-\mathrm{s}$ have $\mathrm{E}_{g}$ in the range of semiconducting materials. The study provides a better understanding on physical properties of coconut shell-derived rGO to further revise synthesis method to improve quality of the obtained rGO.
\end{abstract}

Keywords: coconut shell; density; porosity; reduced graphene oxide.

*Corresponding author: retno.asih@its.ac.id, darminto@physics.its.ac.id

http://dx.doi.org/10.12962/j24604682.v16i2.6712

2460-4682 (CDepartemen Fisika, FSAD-ITS

\section{INTRODUCTION}

Carbon compounds derived from biomass have become potential alternatives to minimize the use of unrenewable mining coals in many industrial applications. Because of its orbital hybridization and bonding nature, carbon has several allotropes that have different physical properties. Graphene, fullerenes and carbon nanotube (CNT) are carbon's allotropes with $\mathrm{sp}^{2}$ hybridization, while diamond is the allotrope with $\mathrm{sp}^{3}$ hybridization [1]. One of the graphene derivatives that has mixed $\mathrm{sp}^{2} / \mathrm{sp}^{3}$ hybridization is reduced graphene oxide ( $\left.\mathrm{rGO}\right)$. rGO has a graphitic structure as that of graphene with some defects, oxygen, and hydrogen on its sheet [2,3]. Thus, rGO can have a wide range of properties which in some cases yield better performance as a chemical sensor [4,5], supercapacitor [6-8], hydrogen storage [9], photovoltaic cells [10,11], and so on. rGO has been prepared from natural resources using several methods including carbonization, pyrolysis and hydrothermal [12-15]. Natural resources provide a new insight to synthesize carbon-based compounds which is environmentally friendly without exploiting mining materials. However, the challenge is that the obtained carbon materials tend to have poor porous structure and vague morphology. Therefore, study on physical properties of the compounds is the key to improve synthesize method applied. It is also necessary as a base to discuss the observed magnetic and electrical properties of the obtained materials.

The biomass of coconut shells is rich in carbon which is about $70 \%$ of the total elements [16]. It also has high hardness and exhibits high volatility and low ash content [17], thus carbon compound derived from coconut shell is expected to bring in the high specific surface area [18]. Moreover, coconut shell is relatively easy to be found as Indonesia is the thirdlargest producer of coconut in the world [19]. In the present study, we prepared the rGO-like phase from a coconut shell. Physical properties of the synthesized rGO (rGO-s) were then compared to those of the commercial rGO (rGO-c) which was prepared from graphite. Variation in physical properties is expected to differ in the observed magnetic and electrical properties. Thus, we also examined the electrical conductivity of the samples. Despite rGO-c has a better porous structure, defined morphology and higher specific surface area than rGO-s, both samples possess the same density, and their gap energy was found to be in the range of semiconducting compounds. Results in this study provide a better understanding of the physical properties of coconut shell-derived rGO to further revise the synthesis method applied to produce rGO with good quality. 


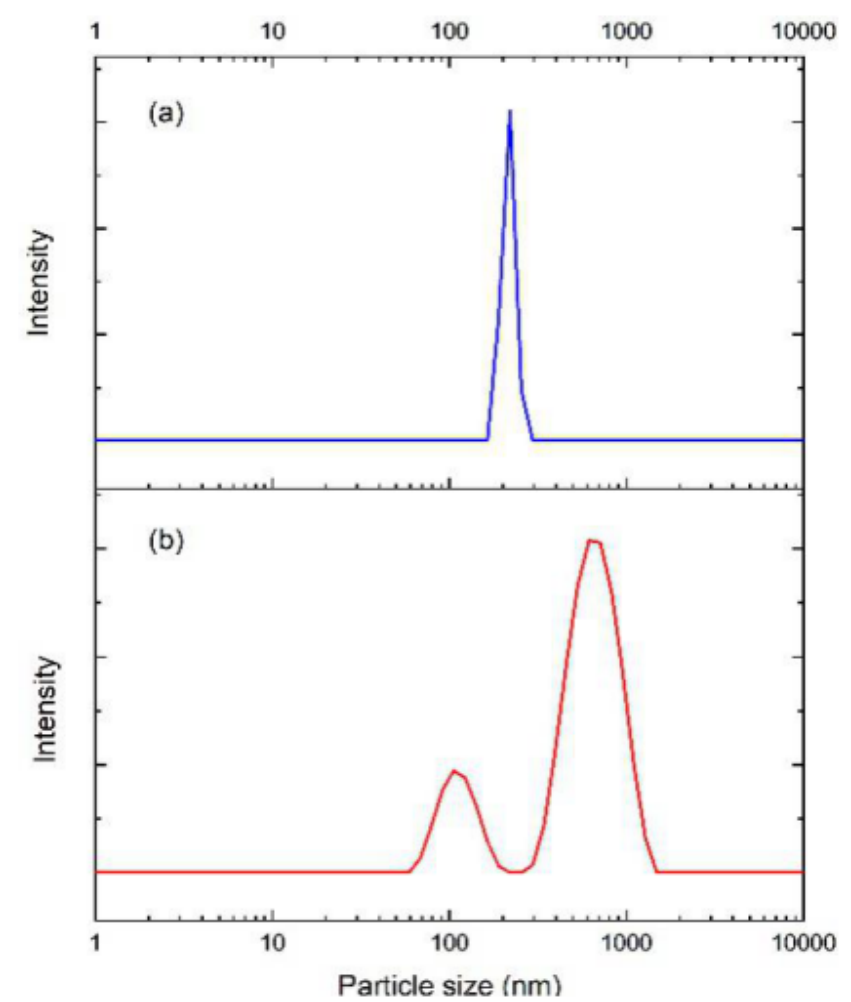

FIG. 1: Particle size distribution of (a) rGO-c and (b) rGO-s analyzed by Particle Size Analyzer (PSA).

\section{EXPERIMENTAL METHODS}

The commercial product of $\mathrm{rGO}$ ( $\mathrm{rGO}-\mathrm{c}$ ) was bought from Graphenea Inc. It was prepared from graphite by the modified Hummer method. Chemical and thermal reduction processes were applied to form rGO from graphite oxide [2, 3]. The synthesized rGO (rGO-c), on the other hand, was prepared from biomass of coconut shell by the carbonization method [21, 23]. The old coconut shell was cleaned by removing husks adhered on its surface. The cleaned shell was carbonized yield in charcoal flakes which were then grounded and sieved to get homogeneous charcoal powders. Furthermore, the powders were sonically exfoliated in the medium of distilled water to form a thinner graphitic layer. Physical properties of the samples, rGO-c, and rGO-s were then examined. Brunauer-Emmett-Teller (BET) theory was applied to identify porously and to measure the specific surface area of the samples. The measurement was carried out using Quantachrome Instruments Nova 1200e Surface Area \& Pore Size Analyzer. The density of the powders was analyzed using a pycnometer. Particle size analyzer (PSA, Zetasizer Nano ZS Malvern) was used to examine particle size distribution of the powders in distilled water. The surface microstructure and its elemental distribution of the powders were also checked using a Scanning Electron Microscope (SEM) with Energy Dispersive X-ray (EDX) spectroscopy. To estimate the gap energy of the samples, optical spectra obtained by the Uv-Vis spectrophotometer (Genesys 10S) in the wavelength range of 200-
TABLE I: Porosity and specific surface area of the samples examined by BET, and density of the samples measured using a pycnometer.

\begin{tabular}{ccccc}
\hline \hline Sample & $\begin{array}{c}\text { P o r o s i t y } \\
\text { Porous } \\
\text { volume (cc/g) }\end{array}$ & $\begin{array}{c}\text { Porous } \\
\text { diameter }(\mathrm{nm})\end{array}$ & $\begin{array}{c}\text { Specific } \\
\text { surface area } \\
\left(\mathrm{m}^{2} / \mathrm{g}\right)\end{array}$ & $\begin{array}{c}\text { Density } \\
\left(\mathrm{g} / \mathrm{cm}^{3}\right)\end{array}$ \\
\hline & & & & \\
rGO-s & 0.18 & 2.44 & 298.53 & 1.95 \\
rGO-c & 1.29 & 10.82 & 475.72 & 1.91 \\
\hline \hline
\end{tabular}

$800 \mathrm{~nm}$ were analyzed using the Tauc Plot method [20]. The electrical conductivity of the samples was determined from V-I data obtained by a four-point probe method.

\section{RESULTS AND DISCUSSION}

Analyses on phase and functional groups presented in the samples have been reported in $[22,23]$. Two broad peaks at $2 \theta$ of about $24^{\circ}$ and $43^{\circ}$ were observed in XRD patterns of the samples, indicating the presence of the rGO-like phase. Spectra of Fourier Transform Infrared (FTIR) also confirmed that both samples have similar functional groups of $\mathrm{C}=\mathrm{C}, \mathrm{C}=\mathrm{O}, \mathrm{C}$ $\mathrm{O}, \mathrm{C}-\mathrm{H}$, and $\mathrm{O}-\mathrm{H}$. However, qualitative analysis of the spectra suggested that rGO-s has a higher percentage of oxygen functionality, which is twice as large as that of rGO-c [23].

Table I summarizes porous characteristics, specific surface area and density of the samples in the powder form. The density of both samples was found to be quite similar, which is 1.91 and $1.95 \mathrm{~g} / \mathrm{cm}^{3}$ for rGO-c and rGO-s, respectively. The volume and diameter of porous presented in $\mathrm{rGO}-\mathrm{c}$ are much larger compared with that of rGO-s. rGO-c has approximately $1.29 \mathrm{cc} / \mathrm{g}$ porous with a diameter of $10.82 \mathrm{~nm}$, while rGO-s has about $0.18 \mathrm{cc} / \mathrm{g}$ porous with a diameter of $2.44 \mathrm{~nm}$. These porous features will directly affect the specific surface area of the samples. The more porous presented in the sample, the larger the surface area will be obtained. rGO-c and rGO-s have a specific surface area of approximately $475 \mathrm{~m}^{2} / \mathrm{g}$ and $298 \mathrm{~m}^{2} / \mathrm{g}$, respectively. Characteristics of large porous and high specific-surface-are are essential in carbon-based materials for their potential applications.

The specific surface area is related to the particle size of the samples. Small particles tend to have a large specific surface area, and vice versa, large particles have a small surface area. Fig. 1 illustrates the particle size distribution of rGO-c and rGO-s examined by PSA. A single peak at $200 \mathrm{~nm}$ was observed in $\mathrm{rGO}-\mathrm{c}$. This indicates that $\mathrm{rGO}-\mathrm{c}$ has homogenous particle-size-distribution. It also implies that the morphology of rGO-c is like thin sheets, thus no other peak signifying a thickness of the sheets can be seen. In the case of rGO-s, two broad peaks are observed at $105 \mathrm{~nm}$ and $615 \mathrm{~nm}$ in the distribution spectrum. It suggests that the particle-size distribution of rGO-s is not quite homogeneous. A small peak at $105 \mathrm{~nm}$ might indicate the thickness of the particle, and a high peak at $615 \mathrm{~nm}$ could be related to a cross-sectional area of the particle. To confirm these suggestions, the surface morphology of 


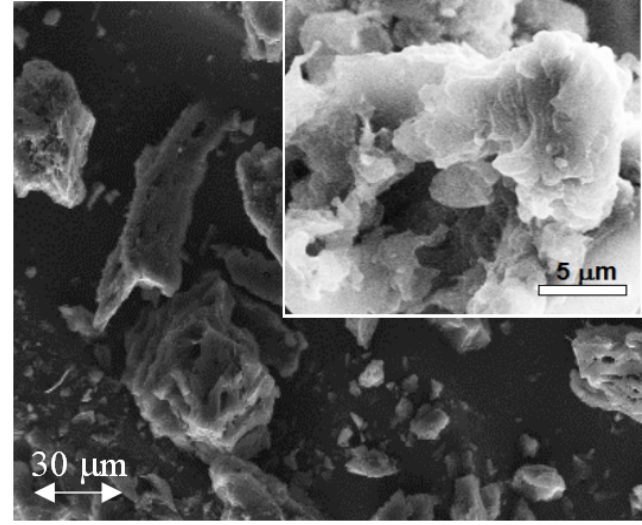

(a) rGO-s

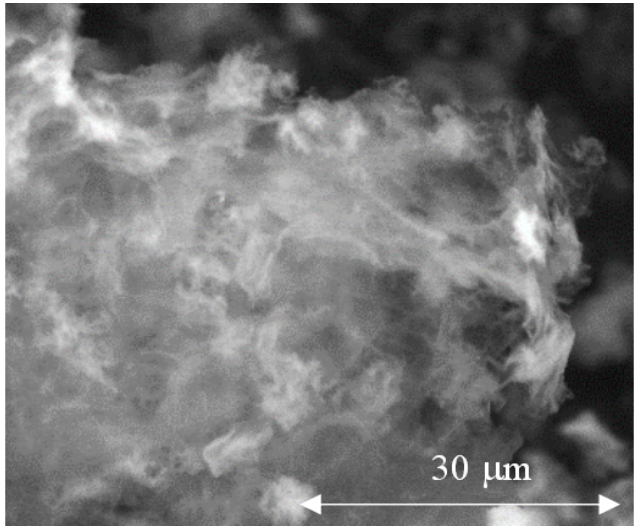

(b) rGO-c

FIG. 2: Surface morphology of (a). rGO-s and (b). rGO-c observed by Scanning Electron Microscope (SEM). Inset in (a) is the close view of a spot in the image.

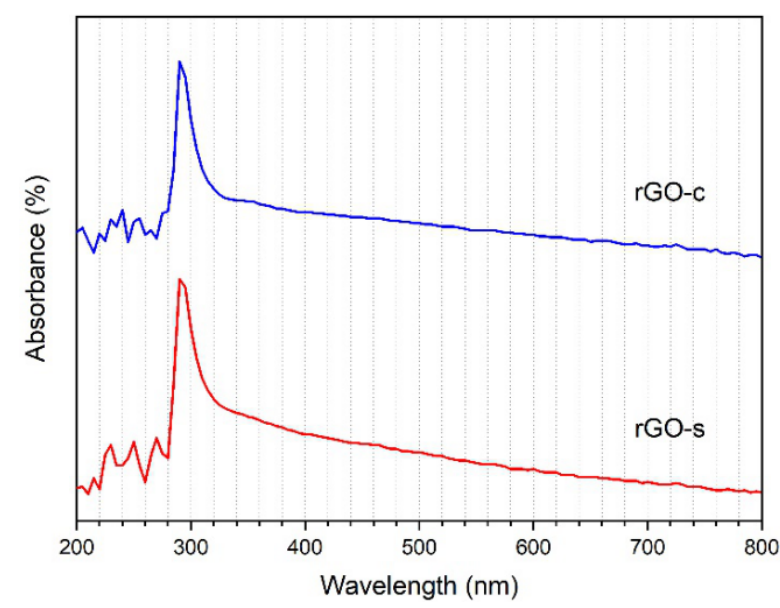

FIG. 3: Optical absorbance spectra of rGO-c and rGO-s obtained from UV-Vis spectroscopy.

the samples was examined, and the results are shown in Fig. 2 .

While transparent thin sheets with homogeneous thickness and size were observed in rGO-c, stacked flakes-like particles with heterogeneous sizes were found in rGO-s. It can be seen in the close view on a spot of the image shown in the inset of Fig. 2(a). From the morphology images, one can notice that there are more porous in rGO-c than rGO-s. Stacking sheets or flakes in rGO-s tends to look denser than those in rGO-c. These somehow consistent with the results suggested by PSA and Surface Area \& Pore Size Analyzer. Elemental analysis by SEM-EDX on the observed surface shows the presence of $\mathrm{C}$ and $\mathrm{O}$ elements in $\mathrm{rGO}-\mathrm{c}$, and $\mathrm{C}, \mathrm{O}$ and $\mathrm{K}$ elements in rGO-s. Relative quantities of each element obtained from SEM-EDX measurement are shown in Table II. Both samples contain a similar amount of $\mathrm{C}$ and $\mathrm{O}$ which are about $80 \mathrm{wt} \%$ and $20 \mathrm{wt} \%$, respectively. A small amount (0.8 wt $\%)$ of potassium $(\mathrm{K})$ is observed in $\mathrm{rGO}-\mathrm{s}$. The existence of $\mathrm{K}$ is predicted since rGO-s was synthesized from the coconut shell.
TABLE II: Relative quantities of elements presented in the surface morphology obtained from SEM with EDX spectroscopy.

\begin{tabular}{lcccc}
\hline \hline Element & \multicolumn{2}{c}{ rGO-s } & \multicolumn{2}{c}{ rGO-c } \\
& (wt\%) & (at\%) & (wt\%) & (at\%) \\
\hline & & & & \\
Carbon (C) & 78.4 & 83.1 & 74.4 & 79.5 \\
Oxygen (O) & 20.8 & 16.6 & 25.6 & 20.5 \\
Potassium (K) & 0.8 & 0.3 & - & - \\
\hline \hline
\end{tabular}

However, this element can be eliminated by heating process and/or dissolution in acid. Thus, a thermal reduction is necessary to prepare a rGO-like phase from biomass in addition to mechanical exfoliation.

The physical features of the samples influence their electrical and magnetic properties. Our previous studies reported that magnetization of the rGO-like phase synthesized from coconut shell strongly depends on defects $[22,23]$. The magnetization enhances when the concentration of defect increases, and this can be tuned by controlling temperature applied in the thermal reduction process [22]. The saturation magnetization of rGO-s is larger than that in rGO-c as rGO-s has more defects compared with rGO-c [23]. In this case, a contribution from magnetic impurities has been subtracted. Here, electrical conductivity $(\sigma)$ of rGO-c was found to be one order larger than that of rGO-s, in which $\sigma$ is about $28 \mathrm{~S} / \mathrm{m}$ and $4 \mathrm{~S} / \mathrm{m}$ for rGO-c and rGO-s, respectively. By evaluating $\sigma$ at the elevated temperature between $30^{\circ} \mathrm{C}$ and $100^{\circ} \mathrm{C}$ with $10^{\circ} \mathrm{C}$ step, we found that gap energy is approximately $0.30 \mathrm{eV}$ for both samples. Furthermore, gap energy was also examined from optical absorption spectra measured by UV-Vis spectrometer.

Fig. 3 displays the absorption spectra of rGO-c and rGO-s solution with the same concentration within the wavelength range of $200-800 \mathrm{~nm}$. The first edge peak at $\sim 230 \mathrm{~nm}$ was observed in both samples. This peak indicates $\pi-\pi^{*}$ electron transition of aromatic C-C. Maximum absorption occurs at the same wavelength of $290 \mathrm{~nm}$ in the samples. This peak implies 
the presence of $n-\pi^{*}$ transition originated from $\mathrm{C}=\mathrm{O}$. By applying the Tauc Plot method [20], plot of photon energy (in $\mathrm{eV}$ ) and $(\alpha \mathrm{h} \nu)^{2}$ (in $\left.\left[\mathrm{eVcm}^{-1}\right]^{2}\right)$, gap energy was estimated to be $0.31 \mathrm{eV}$ and $0.37 \mathrm{eV}$ for rGO-s and rGO-c, respectively. These values agree with those calculated from V-I data and are in the range of gap energy of semiconducting materials. It indicates that the obtained rGO-like phase can be potentially applied in technologies required semiconductor compounds, such as solar cell.

\section{SUMMARY}

Investigation on physical properties has been done on reduced graphene oxide (rGO)-like phase prepared from biomass of coconut shell (rGO-s) and the commercial product (rGO-c). The density of rGO-c and rGO-s was found to be $\sim 1.9 \mathrm{~g} / \mathrm{cm}^{3}$. Analysis of porous characteristics shows that $\mathrm{rGO}-\mathrm{c}$ has more porous of about $1.3 \mathrm{cc} / \mathrm{g}$ with a diameter of $10.8 \mathrm{~nm}$ compared to rGO-s which has $0.2 \mathrm{cc} / \mathrm{g}$ porous with a diameter of $2.4 \mathrm{~nm}$. The specific surface area in rGOc was also obtained much larger than that of rGO-s. rGOc and rGO-s have a specific surface area of $\sim 298 \mathrm{~m}^{2} / \mathrm{g}$ and $\sim 475 \mathrm{~m}^{2} / \mathrm{g}$, respectively. Examinations on particle size and surface morphology show that rGO-c has homogenous particles which consist of transparent thin sheets, while rGO-s has rather heterogeneous particles that look like dens stacked sheets. Moreover, the presence of $\mathrm{C}$ and $\mathrm{O}$ was confirmed at the observed morphology. These physical features were then found to influence the obtained electrical conductivity of the samples. rGO-c has higher conductivity than rGO-s. Estimation on gap energy $\left(\mathrm{E}_{g}\right)$ from both data, electrical conductivity, and optical absorption spectra, indicates that $\mathrm{rGO}-\mathrm{c}$ and rGO-s have $\mathrm{E}_{g}$ in the range of semiconducting materials. To enhance the porous and specific surface area, thermal reduction followed by chemical and mechanical exfoliations is suggested to prepare rGO from coconut shell.

\section{Acknowledgments}

This research was partially supported by the PDUPT research grant 2019. Authors would like to thank the Research Center of Institut Teknologi Sepuluh Nopember for the supports of material characterizations.
[1] J. Tuček, P. Błoński, J. Ugolotti, A. K. Swain, T. Enoki, R. Zbořil, "Emerging chemical strategies for imprinting magnetism in graphene and related 2D materials for spintronic and biomedical applications", Chem. Soc. Rev., vol. 47, pp. 38993990, 2018.

[2] S.K. Sarkar, K.K. Raul, S.S. Pradhan, S. Basu, and A. Nayak, "Magnetic properties of graphite oxide and reduced graphene oxide", Phys. E Low-Dimesional Syst. Nanostruct., vol. 64, pp. 78-82, 2014.

[3] S. Qin, X. Guo, Y. Cao, Z. Ni and Q. Xu, "Strong ferromagnetism of reduced graphene oxide", Carbon, vol. 78 pp. 559 $565,2014$.

[4] I-D. Kim, S-J. Choi, H-J. Cho, "Graphene-based composite materials for chemical sensor application, electrospinning for high performance sensors", in: A. Macagnano, E. Zampetti, E. Kny (Eds.). Cham: Springer International Publishing, 65-101, 2015.

[5] C.R. Minitha, V.S. Anithaa, V. Subramaniam, R.T.R. Kumar, "Impact of oxygen functional groups on reduced graphene oxide-based sensors for ammonia and toluene detection at room temperature", ACS Omega, vol. 3, pp. 4105-4112, 2018.

[6] J. Ma, T. Xue, and X. Qin, "Sugar-derived Carbon/Graphene Composite Materials as Electrodes for Supercapacitors", Electrochimica Acta, vol 115, pp. 566-572, 2014.

[7] S. Rasul, A. Alazmi, K. Jaouen, M.N. Hedhili, P.M.F.J. Costa, "Rational design of reduced graphene oxide for superior performance of supercapacitor electrodes", Carbon, vol. 111, pp. 774-781, 2017.

[8] S. Jayaraman, et al., "Li-ion vs. Na-ion capacitors: A performance evaluation with coconut shell derived mesoporous carbon and natural plant based hard carbon", Chemical Engineering Journal, vol. 316, pp. 506-513, 2017.

[9] J.M. Kim, et al., "Energy storage of thermally reduced graphene oxide", International Journal of Hydrogen Energy, vol. 39, pp. 3799-3804, 2014
[10] M. Czerniak-Reczulska, A. Niedzielska, A. Jdrzejczak, "Graphene as a material for solar cells applications", Advances in Materials Sciences, vol. 15, p. 67, 2015.

[11] D. Barpuzary, and M. Qureshi, "Graphene filled polymers in photovoltaic, graphene-based polymer nanocomposites in electronics", in: K.K. Sadasivuni, D. Ponnamma, J. Kim, S. Thomas (Eds.). Cham: Springer International Publishing, 157191, 2015.

[12] S.S. Shams, L.S. Zhang, R. Hu, R. Zhang, J. Zhu, "Synthesis of graphene from biomass: A green chemistry approach", Mat. Lett., vol. 161, pp. 476-479, 2015.

[13] A. Jain, R. Balasubramanian, M.P. Srinivasan, "Production of high surface area mesoporous activated carbons from waste biomass using hydrogen peroxide-mediated hydrothermal treatment for adsorption applications", Chemical Engineering Journal, vol. 273, pp. 622-629, 2015.

[14] S. Jung, Y. Myung, B.N. Kim, I.G. Kim, I.-K. You, T. Kim, "Activated biomass-derived graphene-based carbons for supercapacitors with high energy and power density", Sci. Reports, vol. 8, p. 1915, 2018.

[15] Y. Liu, J. Chen, B. Cui, P. Yin, C. Zhang, ”Design and preparation of biomass-derived carbon materials for supercapacitors: A review", J. of Carbon Research, vol. 4. p. 53, 2018.

[16] H.M. Mozammel, O. Masahiro and S.C. Bhattacharya, "Activated charcoal from coconut shell using $\mathrm{ZnCl}_{2}$ activation", Biomass and Bioenergy, vol. 22, pp. 397-400, 2002.

[17] M. Yalcin and A.I. Arol, "Gold cyanide adsorption characteristics of activated carbon of non-coconut shell origin", Hydrometallurgy, vol. 63, pp. 201206, 2002.

[18] Z. Liu and F.-S. Zhang, "Removal of copper(II) and phenol from aqueous solution using porous carbons derived from hydrothermal chars", Desalination, vol. 267, pp. 101106, 2011.

[19] D.D. Hendaryati, and Y. Arianto, 'Tree Crop Estate Statistics of Indonesia 2015-2017", Directorate General of Estate Crops, 
1-98, 2017.

[20] J. Tauc, "Optical properties and electronic structure of amorphous Ge and Si", Materials Research Bulletin, vol. 3, pp. 3746, 1968.

[21] Darminto, M.A. Baqiya, R. Asih, "Pengembangan bahan karbon dari biomassa", ITS press, Surabaya, 2018.

[22] Darminto, et al.,"Enhanced magnetism by temperature induced defects in reduced graphene oxide prepared from coconut shells”, IEEE Trans. Mag., vol. 54, pp. 1-5, 2018.

[23] R. Asih, et al.,'Comparative study on magnetism of reduced graphene oxide (rGO) prepared from coconut shells and the commercial product", Mat. Sci. Forum, vol. 966, pp. 290-295, 2019. 